\title{
TRANSICIÓN Y ADAPTACIÓN A LOS ESTUDIOS UNIVERSITARIOS DE LOS DEPORTISTAS DE ALTO NIVEL: LA COMPLEJA RELACIÓN ENTRE APRENDIZAJE Y PRÁCTICA DEPORTIVA
}

\section{TRANSITION AND ADAPTATION TO UNIVERSITY STUDIES OF HIGH-LEVEL ATHLETES: THE COMPLEX RELATIONSHIP BETWEEN LEARNING AND SPORTS}

\author{
Pedro Ricardo Álvarez Pérez ${ }^{1}$ \\ David Pérez-Jorge \\ David López Aguilar \\ Ana Isabel González Herrera \\ Universidad de La Laguna \\ La Laguna, España
}

\section{RESUMEN}

El objeto de este estudio es abordar la compleja relación entre proceso formativo y práctica deportiva en estudiantes universitarios deportistas de alto nivel. Las dificultades para conciliar estos dos tipos de actividades, hace necesario un análisis de las condiciones bajo las cuales estos estudiantes llevan a cabo su formación universitaria y de las ayudas que demandan para la alternancia de ambos procesos, sin tener que renunciar a ninguno de ellos. Siguiendo un método descriptivo y a través de la aplicación de una prueba de cuestionario a una muestra de 81 estudiantes deportistas de alto nivel, se recogió información en relación a cuatro factores clave: la orientación preuniversitaria recibida por los estudiantes deportistas de alto nivel, el proceso de elección de los estudios universitarios, la adaptación a los estudios universitarios y los apoyos y recursos recibidos en la universidad para armonizar estudios y deporte. Los resultados indican que son diversas las variables que condicionan el desarrollo paralelo de estas dos actividades (tiempo, motivación, competencias básicas, exigencias formativas, etc.). Del estudio se deriva la necesidad de personalizar los procesos de enseñanza aprendizaje y de poner en práctica

\footnotetext{
${ }^{1}$ Correspondencia: Pedro Ricardo Álvarez Pérez. Avenida Universidad s/n. 38206. San Cristóbal de La Laguna. Islas Canarias. España. Correo-e: palvarez@ull.es; web: http://www.gufoi.ull.es
} 
medidas orientadoras de carácter preventivo, que den respuesta a las necesidades educativas específicas de estos estudiantes.

Palabras clave: Estudios y deporte, tutoría para estudiantes deportistas, transiciones académicas, toma de decisiones, proceso de aprendizaje.

\section{ABSTRACT}

The purpose of this study is to address the complex relationship between learning process and college sports in high level athletes. The difficulty of reconciling these two types of activities requires an analysis of the conditions under which these students pursue their university education and demanding aid for the alternation of the two processes without having to give up any of them. Following a descriptive method and through the application of a questionnaire to test a sample of 81 senior student-athletes, collected information relating to four key factors: the orientation preuniversity students received high-level athletes, the process choice of college, adjusting to college and received support and resources to harmonize university studies and sports. The results indicate that there are many variables that determine the parallel development of these two activities (time, motivation, basic skills, training requirements, etc.). The study is the need to personalize the teaching and learning processes and to implement preventive measures guiding that respond to the specific educational needs of these students.

Key Words: Studies and sports, tutoring for student athletes, academic transitions, decision making, learning process.

\section{Introducción}

Son muchos los estudiantes universitarios que alternan la práctica deportiva de alto nivel, con una formación académica y científica a través de la cual obtienen la cualificación necesaria para acceder posteriormente al mercado de trabajo y desarrollarse profesionalmente. Sin embargo, resulta complejo encontrar un equilibrio entre estas dos actividades y son muchos los estudiantes que terminan por abandonar alguna de ellas. En el caso de los estudios, el proceso de aprendizaje se ve con frecuencia comprometido, al no poder seguir los estudiantes deportistas de alto nivel el mismo ritmo que el resto de compañeros y compañeras. La asistencia regular a clase, la presencia física en los períodos de prácticas, la elaboración de los trabajos en grupo, las prácticas externas, la realización de los exámenes en el día y hora establecidos por los Centros y Facultades, etc. son aspectos claves de la vida universitaria en el modelo del Espacio Europeo de Educación Superior (EEES) que muchos de los alumnos y alumnas deportistas de alto nivel no pueden cumplir debido a las exigencias deportivas.

La práctica deportiva para los estudiantes no puede ser considerada una cuestión elitista. Por el contrario, se deberían articular las medidas necesarias para que la actividad deportiva sea una parte del proceso de formación y de desarrollo del alumnado, puesto que a través de la misma se potencian muchas habilidades y competencias genéricas de relevancia para la vida en sociedad. La práctica deportiva ofrece a los jóvenes la oportunidad de desarrollar habilidades sociales, aprender a trabajar en equipo, afrontar situaciones de resolución de problemas y toma de decisiones, planificarse para alcanzar metas, adquirir responsabilidad en el logro de objetivos, etc. Todas estas son competencias genéricas de relevancia no solo para la práctica deportiva, sino 
para otros planos de la vida personal, social y laboral de las personas (Rychen y Salganik, 2001; Corominas, 2001; Rodríguez, 2007; Riesco, 2008).

Cada vez más se pone de manifiesto que el acceso al mercado de trabajo pasa por el dominio de una diversidad de competencias; no solo conocimientos y habilidades específicas en un campo científico determinado, sino también competencias genéricas y transversales necesarias para transitar y moverse en el complejo entramado sociolaboral (Villa y Benzanilla, 2002; García, 2006; Poblete y col., 2007). En este sentido señala Blanco (2009:15) que "existen ciertas competencias generales que bien podrían ganarse el atributo de ser universales porque el valor que aportan al estudiante contribuye a su desarrollo integral, a su enriquecimiento formativo como ciudadano, como profesional y en definitiva, como ser humano ubicado en la era que nos toca vivir". La propia OCDE (2005) en su proyecto "definición y selección de competencias" (DESECO) considera que el dominio de una competencia ha de permitir resolver situaciones complejas poniendo en juego diversas habilidades, recursos y actitudes.

La sociedad actual exige que las personas tengan capacidad de adaptarse de manera permanente a los entornos complejos. Y esto exige, sin duda, que los jóvenes adquieran a lo largo de la vida las competencias genéricas para su desarrollo como personas y como profesionales. De ahí que el enfoque de una enseñanza integrada en la educación superior se haya convertido en un objetivo básico recogido en muchas de las normativas de esta etapa. En el Real Decreto $55 / 2005$ se señala que las enseñanzas de grado deben "propiciar la consecución por los estudiantes de una formación universitaria que aúne conocimientos generales básicos y conocimientos transversales relacionados con su formación integral, junto con los conocimientos y capacidades específicos orientados a su incorporación al ámbito laboral". Asimismo, el Real Decreto 1044/2003 establece que en el Suplemento Europeo al Título que deben expedir las universidades se tiene que recoger información precisa y detallada sobre "los estudios cursados, los resultados obtenidos, las capacidades profesionales adquiridas y el nivel de su titulación". Finalmente, en el Real Decreto 861/2010 que regula las enseñanzas oficiales universitarias, cuando se refiere a las titulaciones de grado, se señala que los estudiantes han de saber "aplicar sus conocimientos a su trabajo o vocación de una forma profesional y poseer las competencias que suelen demostrarse por medio de la elaboración y defensa de argumentos y la resolución de problemas dentro de su área de estudio".

Para adquirir y desarrollar muchas de estas competencias que habilitan para el desarrollo profesional, es necesario que los jóvenes reciban la formación avanzada necesaria. En el caso de los deportistas de alto nivel que deciden cursar estudios universitarios, se tendrían que contemplar diversas medidas de atención y apoyo que den una respuesta a las necesidades específicas de cada uno, para que puedan alcanzar los objetivos formativos que les han de capacitar para la vida laboral. Sin el asesoramiento adecuado, muchos estudiantes deportistas corren el riesgo de abandonar los estudios, dado que en muchos casos es la única salida que encuentran para liberarse de la presión y el estrés que provoca la suma de tareas y las obligaciones de ambos tipos de actividades, lo cual puede afectar de manera decisiva en el futuro personal y social de estos jóvenes (Palou, Ponsenti, Gili, Borras y Vidal, 2005).

La conclusión que se puede extraer de todo esto es que se deben mejorar las condiciones de acceso, de orientación y de formación para que los jóvenes deportistas puedan realizar sus estudios universitarios. Una acción orientadora que forme para la autonomía, para la definición de la identidad personal, para canalizar los intereses a través de un proyecto formativo y profesional, para mantener una actitud positiva hacia la formación continua, para el análisis crítico de la realidad y para la adaptación a los cambios que se suceden de manera vertiginosa en el conjunto de la sociedad (Álvarez, 2002; Álvarez y Lázaro, 2002). La tutoría constituye actualmente un factor estratégico de gran potencial para la mejora de la calidad del nuevo modelo educativo del EEES, puesto que a través de sus acciones se puede lograr una mejora en los procesos de transición y 
adaptación del alumnado, la optimización del proceso formativo, la prevención del abandono de los estudios y la mejora en los procesos de desarrollo profesional. Por eso, a nivel europeo, se han establecido estándares para asegurar la calidad en el EEES, y se ha regulado que las instituciones deberán garantizar los recursos para que el apoyo del aprendizaje del alumnado sea el adecuado y apropiado para cada programa de formación.

De acuerdo con esta perspectiva, en este trabajo se lleva a cabo un análisis de necesidades en relación a la armonización entre proceso de aprendizaje y práctica deportiva. Concretamente se pretende determinar qué problemas, qué necesidades y qué demandas plantean los estudiantes universitarios deportistas de alto nivel para poder compaginar estudios y deporte. Los datos recogidos servirán de referencia para planificar acciones tutoriales y orientadoras que les ayuden a conciliar ambos procesos, organizando y conduciendo su proyecto personal sin que se produzcan situaciones de fracaso y/o abandono de los estudios.

\section{Conciliación entre estudios y práctica deportiva}

El acceso a la educación superior de los estudiantes deportistas de alto nivel supone un cambio importante en cuanto al nivel de exigencia y dedicación, constituyendo un momento clave en el que se debe contar con la ayuda y el asesoramiento necesario para que la transición y la adaptación se produzca de manera satisfactoria (Rodríguez, Fita y Torrado, 2004; Álvarez, Figuera y Torrado, 2011). El proceso de integración a la vida universitaria de los estudiantes deportistas de alto nivel, no solo se complejiza debido a las dificultades derivadas de la doble actividad (estudios y deporte), sino por las propias características del nuevo modelo formativo que surge con la creación del EEES. Efectivamente, el EEES impone un nuevo modelo de formación, una metodología diferente, nuevas fórmulas de evaluación, diferentes relaciones interpersonales entre profesor-alumno y sobre todo, se impone el aprendizaje sobre la enseñanza, al considerarse al estudiante como principal protagonista del proceso formativo y gestor de su proceso de aprendizaje autónomo (Benito y Cruz, 2005, Sánchez y Zubillaga, 2005).

Afrontar con éxito este proceso de aprendizaje autónomo exige no solo compromiso, esfuerzo y dedicación, sino también tiempo para abordar las distintas tareas y cumplir con los requisitos del seguimiento y la evaluación. Por eso, las dificultades para cumplir con las exigencias del proceso formativo, los inconvenientes para compaginar tareas de diferente naturaleza, la falta de recursos, la escasa motivación, la falta de metas claras o la escasez de tiempo para atender a todas las actividades, se encuentran muchas veces en la base de situaciones de prolongación o de fracaso y/o abandono de los estudios que viven los estudiantes deportistas de alto nivel (Márquez, Salguero y Tuero, 2003; Morillas, Gamito, Gómez, Sánchez y Valiente, 2004). Estudios como el realizado por González, Álvarez, Cabrera y Bethencourt (2007) ponen de relieve la trascendencia del problema del abandono. En la investigación que realizaron con una muestra de 163 estudiantes de tres titulaciones universitarias diferentes (Pedagogía, Matemáticas y Trabajo Social) para identificar factores asociados a la probabilidad de deserción y prolongación de los estudios, obtuvieron un porcentaje del $28 \%$ de abandono de los estudios en los dos primeros años. Entre los motivos del abandono destacaron la desmotivación y la falta de ilusión por la carrera, bien porque no resultó ser lo que esperaban o porque era muy difícil.

Todas estas dificultades y necesidades han llevado a que en los últimos tiempos se hayan empezado a introducir distintas medidas encaminadas a dar una respuesta adecuada a las necesidades y características específicas de estos estudiantes. La aprobación del Estatuto del Estudiante Universitario (Real Decreto 1791/2010), ha supuesto un avance importante para que se empiece a considerar la atención específica que requieren los estudiantes deportistas de alto 
nivel. Tanto en el artículo 61 (principios generales), como en el 62 (actividad física y deportiva de los estudiantes) del Capítulo XII (de la actividad deportiva de los estudiantes), se insiste en la idea de que la actividad deportiva es un "componente de la formación integral del estudiante", por lo que las universidades deben facilitar "la compatibilidad de la actividad académica y deportiva de los estudiantes" y la puesta en funcionamiento de "los sistemas de orientación y seguimiento" que hagan posible la armonización de la formación con la práctica deportiva.

Para cumplir con estas exigencias que establece la normativa, no solo hay que mejorar el apartado de infraestructura, creando instalaciones que permitan una actividad deportiva acorde con los requisitos de la alta competición, sino que se deben introducir los apoyos académicos necesarios para que el proceso formativo de estos jóvenes sea satisfactorio. $Y$ una de las medidas académicas importantes para hacer frente a las dificultades de estos estudiantes y dar respuestas a las necesidades específicas de los mismos de forma que puedan desarrollar en paralelo su carrera deportiva y su formación académica universitaria, es la tutoría universitaria (Rodríguez, 2004; Álvarez, 2008; Martínez, 2009). A través de la función tutorial se pretende fomentar los procesos de integración y adaptación al sistema formativo de los estudiantes deportistas de alto nivel, llevar a cabo un seguimiento personalizado de su proceso de aprendizaje que contribuya a un mejor aprovechamiento académico y personal (la mejor utilización de los recursos disponibles, la mejora de las aptitudes personales, las técnicas de aprendizaje, etc.) y facilitar la toma de decisiones respecto a su formación y planificación de su proyecto académicoprofesional (Campos, Ríes y Castillo, 2011). Es por tanto una medida para compensar las dificultades que tienen estos estudiantes y para fomentar principios fundamentales como la equidad o la igualdad. Como señala Arnaiz (2002), se debe ejercer en la enseñanza el principio de igualdad y el respeto a la diversidad al que todo ser humano tiene derecho, lo que implica el desarrollo de estrategias de enseñanza aprendizaje que personalicen la educación en un contexto formativo plural y para todos. El problema estriba en que muchos docentes carecen de los recursos y de la formación necesaria para poder realizar adaptaciones metodológicas, para dar una respuesta didáctica específica e individualizada. Esa es una debilidad de la institución universitaria que se debería mejorar para que "el profesorado organice y desarrolle sus materias con metodologías adecuadas como para permitir la participación de los estudiantes con necesidades educativas específicas, en igualdad de condiciones que el resto del alumnado" (Álvarez, 2012:23).

Y en pos del logro de este objetivo, la intervención tutorial en la enseñanza universitaria se ha convertido en una estrategia fundamental para personalizar y singularizar la enseñanza, aplicando métodos de enseñanza flexibles que contemplen la diversidad de situaciones y den respuesta a las necesidades diversas del alumnado, ya que como señala la UNESCO, se tendría que promover una perspectiva inclusiva de la educación, para brindar oportunidades de aprendizaje a todo el alumnado. Se destaca la importancia de dar "respuesta a la diversidad de las necesidades de todos los alumnos a través de la creciente participación en el aprendizaje, las culturas y las comunidades, y de la reducción de la exclusión dentro y desde la educación. Implica cambios y modificaciones en los enfoques, las estructuras, las estrategias, con una visión que incluye a todos los niños de la franja etaria adecuada y la convicción de que es responsabilidad del sistema regular educar a todos los niños" (UNESCO, 2005:13)".

En el contexto universitario del Estado español se recoge de alguna manera este principio básico, cuando en el Real Decreto 1393/2007 de ordenación de las enseñanzas universitarias oficiales (corregido por el Decreto 861/2010) se señala que la finalidad de los títulos de grado es facilitar el logro por parte de los estudiantes de una formación universitaria que reúna conocimientos generales básicos y conocimientos transversales relacionados con su formación integral, junto con los conocimientos y capacidades específicas orientadas a su incorporación al mundo laboral. En el mismo Reglamento se establece que los centros universitarios deberán atender los procesos de acceso y apoyo al alumnado mediante sistemas de información previa a 
la matriculación y procedimientos de acogida y orientación de los estudiantes de nuevo ingreso para facilitar su incorporación a la universidad y sistemas de apoyo y orientación de los estudiantes una vez matriculados.

En el caso de estos estudiantes que compaginan estudios y deporte, la tutoría se ve como una estrategia pedagógica para apoyar y asesorar a los estudiantes en su proceso de integración, de desarrollo y de formación. Pretende potenciar las capacidades de cada estudiante y ayudarles a superar las dificultades que aparecen a lo largo del proceso educativo. Por ello y dado que la tutoría se presenta como un espacio en íntima conexión con la enseñanza, no hay un lugar marcado y delimitado para su desarrollo. La tutoría debería servir también para que el profesorado oriente y valore con el alumnado sus progresos y también sus dificultades, buscando cauces de mejora (Álvarez, 2002; Zabalza, 2002). Esta interacción entre el alumnado y el tutor supone compartir experiencias, conocimientos y métodos de aprendizaje a través de un trabajo cooperativo y dialógico, donde el estudiantado revisa, organiza y se proyecta hacia nuevos conocimientos que le permiten asumir otros retos, incorporándolos a su proyecto formativo y profesional. Para el alumnado que tiene dificultades, resulta tremendamente importante que se valore, desde una perspectiva constructiva y formativa, en qué cosas ha fallado, qué errores de comprensión y perspectiva ha tenido, cómo tendría que haber orientado la tarea, cómo mejorar su proceso de aprendizaje, qué competencias debería reforzar, cómo afrontar el proceso de aprendizaje autónomo, qué estrategias y recursos de aprendizaje utilizar, qué documentos y bibliografía le podrían resultar de interés, etc. Sin este tipo de ayuda personalizada, sin esta colaboración para resolver las dificultades relacionadas con el proceso de enseñanza aprendizaje, muchos estudiantes tendrán dificultades para gestionar la alternancia y lograr las metas académicas.

\section{Método}

\section{Objetivos}

El objetivo general de esta investigación fue valorar cómo afrontan el acceso a la enseñanza universitaria los estudiantes deportistas de alto nivel y qué dificultades tienen para adaptarse y compaginar el proceso de enseñanza aprendizaje con la práctica deportiva. Se planteó como hipótesis inicial que estos estudiantes, con unas necesidades educativas específicas, se enfrentan a diversidad de problemas para compaginar estudios y deporte, lo que les puede conducir al abandono de los estudios. Para profundizar en torno a este problema de armonización entre estudios y deporte, se plantearon los siguientes objetivos específicos:

- Analizar la orientación recibida por los estudiantes deportistas de alto nivel en las etapas previas a la enseñanza universitaria.

- Conocer cómo afrontan la elección de estudios y al acceso a la educación superior.

- Analizar las dificultades para adaptarse a los estudios universitarios.

- Conocer los recursos y apoyos que reciben en la universidad para conciliar aprendizaje y práctica deportiva.

- Valorar las demandas que plantean para una mejor conciliación entre estudios y deporte.

La identificación de las dificultades a las que se enfrentan los estudiantes deportistas de alto nivel, permitirá la puesta en práctica de medidas de apoyo que compensen las situaciones de desigualdad y permitan el logro de las metas académicas. Concretamente, el análisis de 
necesidades y los resultados que se obtengan de este estudio, constituirá el referente para la elaboración de propuestas concretas de actuación educativa y orientadora, que sirva para prevenir posibles situaciones de prolongación, fracaso o abandono de la formación.

\section{Participantes}

Se estableció que los participantes en este estudio fueran estudiantes universitarios deportistas de alto nivel, que estuvieran cursando alguna titulación de educación superior en la Universidad de La Laguna en el momento de la realización del estudio. Los requisitos para considerar a un estudiante deportista de alto nivel fueron: practicar un deporte reconocido por el Consejo Superior de Deportes (CSD - Ministerio de Educación, Cultura y Deporte), entrenar un mínimo de ocho horas semanales y competir regularmente a nivel nacional o regional. La selección de la muestra se realizó mediante un procedimiento de muestreo causal o intencional, con el fin de acceder a los deportistas universitarios que reunieran las características previamente establecidas.

El número total de estudiantes universitarios deportistas de alto nivel, como se recoge en la tabla 1, fue de 81. El rango de edad de los participantes se situó entre los 18 y los 34 años $(\bar{x}=21,62 ; S d=2,96)$. En cuanto al sexo, el $59,3 \%$ del alumnado eran hombres y el $40,7 \%$ mujeres. El curso medio que se encontraban realizando era cuarto $(\bar{x}=3,85)$ y se matriculaban en un promedio de 8 asignaturas anuales $(\bar{x}=8,16)$. En cuanto a la modalidad deportiva, los estudiantes participantes practicaban dieciséis tipos de actividades deportivas diferentes, tal como figura también en la tabla 1.

TABLA 1. Características de los participantes

\begin{tabular}{|c|c|c|}
\hline Edad & \multirow{2}{*}{\multicolumn{2}{|c|}{ Rango de Edad: $18-34$ años; $\bar{x}=21,62$ años; $S d=2,96$ años }} \\
\hline Sexo & Hombres=59,3\%; Mujeres $=40,7 \%$ & \\
\hline $\begin{array}{l}\text { Número de asignaturas } \\
\text { matriculadas por curso } \\
\text { académico }\end{array}$ & & $\bar{x}=8,16 ; S d=3,60$ \\
\hline \multirow{8}{*}{ Deporte practicado } & Natación: 8,6\% $(\mathrm{n}=7)$ & Salvamento y socorrismo deportivo: $3,7 \%(n=3)$ \\
\hline & Atletismo: $28,4 \%(n=23)$ & Squash: $1,2 \%(n=1)$ \\
\hline & Voleibol: $4,9 \%(n=4)$ & Frontenis: $3,7 \%(n=3)$ \\
\hline & Vela: $9,9 \%(n=8)$ & Tenis de mesa: $3,7 \%(n=3)$ \\
\hline & Baloncesto: $9,9 \%(n=8)$ & Triatlón: $1,2 \%(n=1)$ \\
\hline & Waterpolo: $4,9 \%(n=4)$ & Fútbol: 9,9\% (n=8) \\
\hline & Judo: $2,5 \%(n=2)$ & Balonmano: $1,2 \%(n=1)$ \\
\hline & Kárate: $6,2 \%(n=5)$ & \\
\hline
\end{tabular}

\section{Procedimiento - instrumentos}

Siguiendo una metodología de carácter descriptivo-exploratorio no experimental, los datos de la investigación se han recogido mediante un cuestionario ad hoc denominado "Cuestionario de Necesidades para Alumnos Deportistas de Alto Nivel" (NADAN), adaptado a las características de la población y a los objetivos que se pretendían alcanzar. 
El instrumento fue sometido a diferentes procedimientos de validación y fiabilidad, con la finalidad de adecuar la prueba a la finalidad que tenía el estudio. Con la versión inicial del cuestionario se realizó una prueba piloto en una sesión presencial con estudiantes que reunían características similares a las de la muestra definitiva $(n=6)$. En esta prueba, 2 evaluadores anotaban las incidencias relacionadas con el contenido (contenido de los ítems, claridad de los enunciados, vocabulario, alternativas de respuesta propuestas, extensión de la prueba, etc.). Además, se realizó una prueba de forma con expertos en metodología de la investigación educativa $(n=3)$ para valorar diferentes aspectos referidos a los tipos de preguntas a utilizar, tipos de escalas, etc. Finalmente, se llevó a cabo una prueba de contenido por parte de expertos en el campo de la educación $(n=3)$ y el deporte $(n=2)$ para analizar diferentes aspectos relacionados con la pertinencia, la claridad, la adecuación de las preguntas, etc.

A partir de este proceso de revisión, se introdujeron en el instrumento varias modificaciones y ajustes en los ítems. Concretamente, se modificaron los enunciados de diferentes preguntas, se introdujeron algunas preguntas nuevas para poder recoger de forma más precisa la información que se precisaba, se añadieron nuevas opciones de respuesta a algunos ítems y se eliminaron tres cuestiones debido a la escasa relevancia que tenían para el estudio. Introducidas estas correcciones, la versión final del instrumento quedó estructurada en torno a cuatro dimensiones: orientación pre-universitaria (I); elección y acceso a los estudios universitarios (II); adaptación a los estudios universitarios (III); apoyos y recursos de la universidad (IV). En relación a estas cuatro dimensiones, se plantearon 53 ítems organizados en escalas de valoración, preguntas dicotómicas con opciones de respuesta abierta, preguntas de respuesta múltiple y preguntas específicas de respuesta abierta.

En cuanto al valor de fiabilidad del instrumento definitivo, la aplicación de la prueba de alfa de Cronbach $(\alpha=.865)$ indicó que existía una alta consistencia interna y un alto grado de interrelación entre los 52 elementos del cuestionario final que reunían características similares.

\section{Análisis e interpretación de los datos}

Una vez administrado el instrumento de recogida de información, los datos fueron analizados a través del programa Statistical Package for the Social Sciences (SPSS 17.0) para el entorno Microsoft Windows 7. Dada la finalidad y los objetivos del estudio, este análisis comprendió un examen propio de los estadísticos descriptivos y de contraste para cada una de las variables estudiadas: frecuencias, porcentajes, coeficientes de fiabilidad ( $\alpha$ de Cronbach), contraste de medias y análisis de correlación (Pearson). Para las preguntas abiertas se utilizó la técnica de análisis de contenido, realizando una categorización de las respuestas ofrecidas por los participantes para cada uno de los ítems.

\section{Resultados}

A continuación se presentan los resultados agrupados en función de las distintas dimensiones analizadas.

\section{Orientación pre-universitaria}

En el momento previo al acceso a la enseñanza superior, los estudiantes deportistas señalaron que tenían información sobre aspectos generales de la universidad, relacionados principalmente con las salidas profesionales que ofrecían las diferentes carreras $(62,2 \%)$, las 
distintas titulaciones existentes (58,0\%) y las ayudas y becas $(51,9 \%)$ de apoyo a la formación. Sin embargo, pocos estudiantes indicaron estar informados sobre algunos aspectos vinculados directamente con el propio proceso formativo, como eran la metodología, los sistemas de evaluación o los programas de orientación al alumnado (ver gráfica 1).

FIGURA 1. Información preuniversitaria

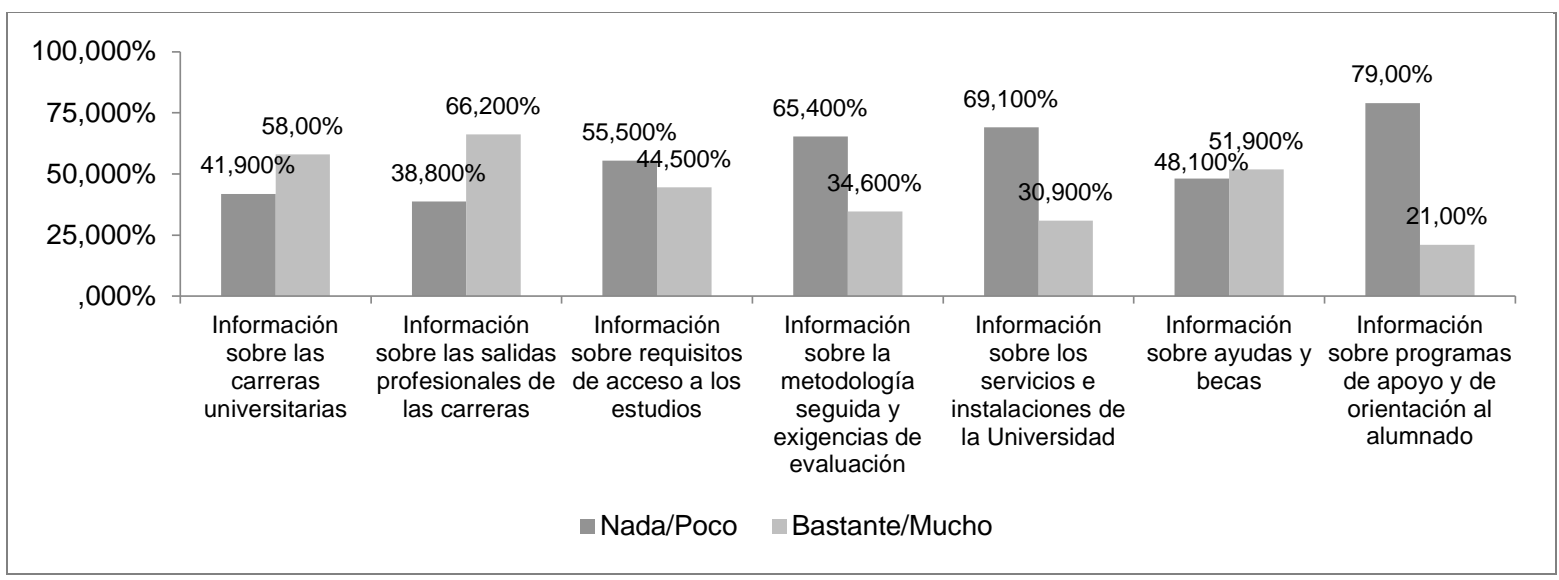

También destacó la escasa información que tenían los estudiantes deportistas referida a algunos aspectos importantes de la vida universitaria, tales como las vías de participación estudiantil (98,6\%), las actividades extraacadémicas que ofrecía la institución (95,8\%), el perfil formativo de las titulaciones (72,2\%) o el plan de estudios de los diferentes grados $(55,6 \%)$. Además, tenían un desconocimiento generalizado sobre algunos aspectos concretos directamente relacionados con la propia práctica deportiva, como las ayudas y becas específicas para los deportistas $(97,2 \%)$ o la existencia de las diferentes instalaciones deportivas de la universidad $(91,7 \%)$.

En cuanto a las personas que les habían proporcionado información sobre la universidad, los estudiantes deportistas hicieron referencia al papel que jugaban los compañeros y las compañeras de clase $(54,4 \%)$ y los familiares $(53,0 \%)$, quienes les informaron sobre las distintas titulaciones ofrecidas en la universidad $(89,9 \%)$ y las posibles salidas profesionales de cada uno de los grados $(59,7 \%)$. No obstante y según los encuestados, cabe destacar la poca relevancia que en este proceso de orientación e información preuniversitaria tuvieron los entrenadores, orientadores escolares, tutores y profesores de secundaria, dado que, a juicio de los encuestados, no ayudaron a clarificar y fortalecer los procesos de toma de decisiones acerca del futuro académico-profesional de los deportistas de alto nivel (ver gráfica 2).

FIGURA 2. Agentes de orientación e información a los estudiantes deportistas de alto nivel

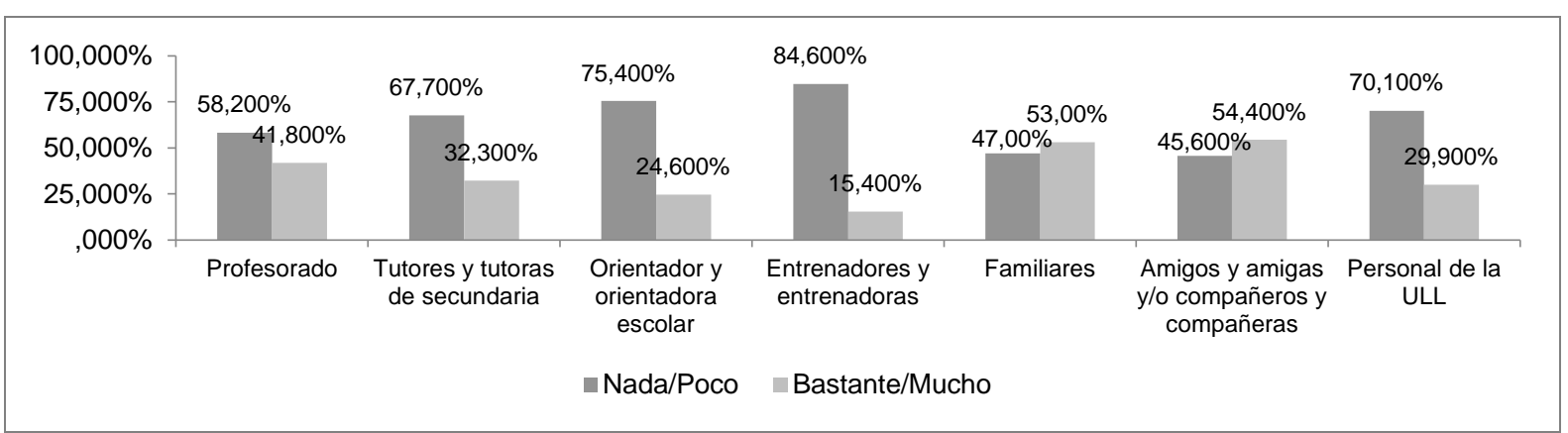




\section{Elección y acceso a los estudios universitarios}

Las valoraciones aportadas por los deportistas de alto nivel, dejan entrever que el alumnado tenía una idea clara y precisa sobre los estudios que quería cursar en el momento de acceder a la universidad (63,0\%). Prueba de ello fue la coincidencia que se encontró entre la titulación que estaban cursando y la primera opción formativa elegida para ingresar en la enseñanza superior $(66,7 \%)$. Este proceso de elección de estudios resultó estar determinado por la información que tenían los deportistas sobre la universidad (servicios, titulaciones, instalaciones deportivas, etc.) $(p<.005)$ y el conocimiento de las salidas laborales que podrían ofrecer las titulaciones $(p<.048)$.

Entre los motivos de elección de estudios, los deportistas destacaron principalmente el gusto por la carrera $(86,4 \%)$, las salidas profesionales de la titulación $(64,2 \%)$, el consejo de personas allegadas (familiares, deportistas, etc.) $(43,2 \%)$ y la estabilidad laboral $(30,9 \%)$.

FIGURA 3. Motivos de elección de estudios universitarios

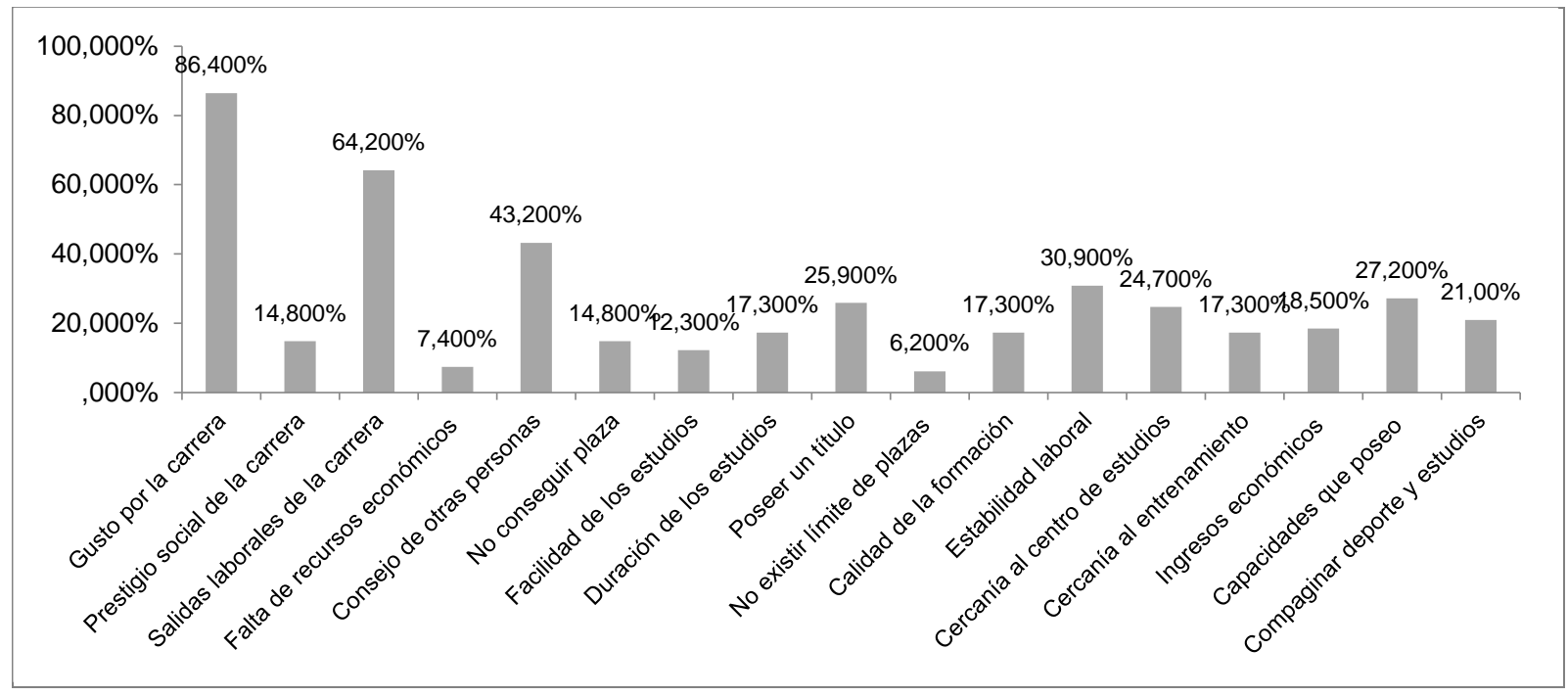

Al analizar la influencia que tenía la práctica deportiva en los procesos de toma de decisiones académicas, se encontraron diferencias significativas $(p<.041)$ entre los motivos de elección de estudios y el deporte practicado, dado que el alumnado prefería elegir una titulación que estuviera vinculada a la práctica deportiva (fisioterapia, maestro especialista en educación física, periodismo, etc.). Además, señalaron que debía ser una titulación que les permitiera realizar sus estudios sin que ello les supusiera la renuncia al deporte $(70,4 \%)$.

Para lograr compatibilizar estas dos actividades, los estudiantes entendían que debían adaptar el proceso de aprendizaje a sus necesidades y posibilidades, aunque para ello tuvieran que dedicar más años de los necesarios para completar su formación (50,6\%). Por tanto, y desde la opinión de los estudiantes, la armonización estudios-deporte $(71,4 \%)$ sería posible siempre y cuando el propio proceso formativo reuniera las condiciones necesarias para flexibilizar el desarrollo académico del alumnado. 


\section{Adaptación a los estudios universitarios}

En el momento de acceso a los estudios universitarios, los deportistas tenían un alto grado de motivación hacia la formación superior (66,3\%). A lo largo de su proceso formativo, los alumnos y alumnas veían confirmadas sus expectativas iniciales en cuanto a los sistemas de evaluación utilizados en la enseñanza universitaria $(58,6 \%)$, las exigencias formativas del profesorado $(57,9 \%)$ y las relaciones con los compañeros y compañeras $(75,0 \%)$ y el profesorado $(70,9 \%)$. Sin embargo, no ocurría lo mismo respecto a la opinión que mostraban sobre la adecuación de los contenidos $(57,2 \%)$ y las prácticas desarrolladas en las materias $(61,4 \%)$. En este sentido, cabe destacar que el estudiantado que tenía una alta motivación hacia los estudios y que además veía confirmadas sus expectativas acerca de la universidad, tenía menos posibilidades de abandonar la formación que realizaban $\left(r_{0}=.447 ; p=.000\right)$, pese a las dificultades y problemas que pudieran advertir en el proceso de armonización entre la práctica deportiva y la enseñanza universitaria.

Las estrategias utilizadas por los deportistas para adaptarse a las exigencias formativas y tener éxito en la enseñanza universitaria, consistieron principalmente en la asistencia regular a clase $(78,5 \%)$ o en solicitar apoyo a los compañeros y compañeras cuando no acudían al aula por motivos deportivos (88,9\%). Sin embargo y aunque el profesorado universitario debería desempeñar la función de guía y orientación del alumnado durante el proceso formativo, desde la perspectiva del alumnado, no ofrecía una respuesta educativa específica ajustada a las necesidades formativas de los deportistas de alto nivel (92,5\%).

En relación a la valoración que hicieron los estudiantes de la orientación recibida durante el proceso de aprendizaje, destacó principalmente la información relacionada con la ubicación física de los espacios $(95,1 \%)$, el perfil de la titulación y el plan de estudios $(84,0 \%)$, las salidas profesionales de la carrera $(76,6 \%)$ y la utilidad de las asignaturas para el desempeño profesional $(61,8 \%)$.

FIGURA 4. Información de los estudiantes deportistas de alto nivel en la enseñanza universitaria

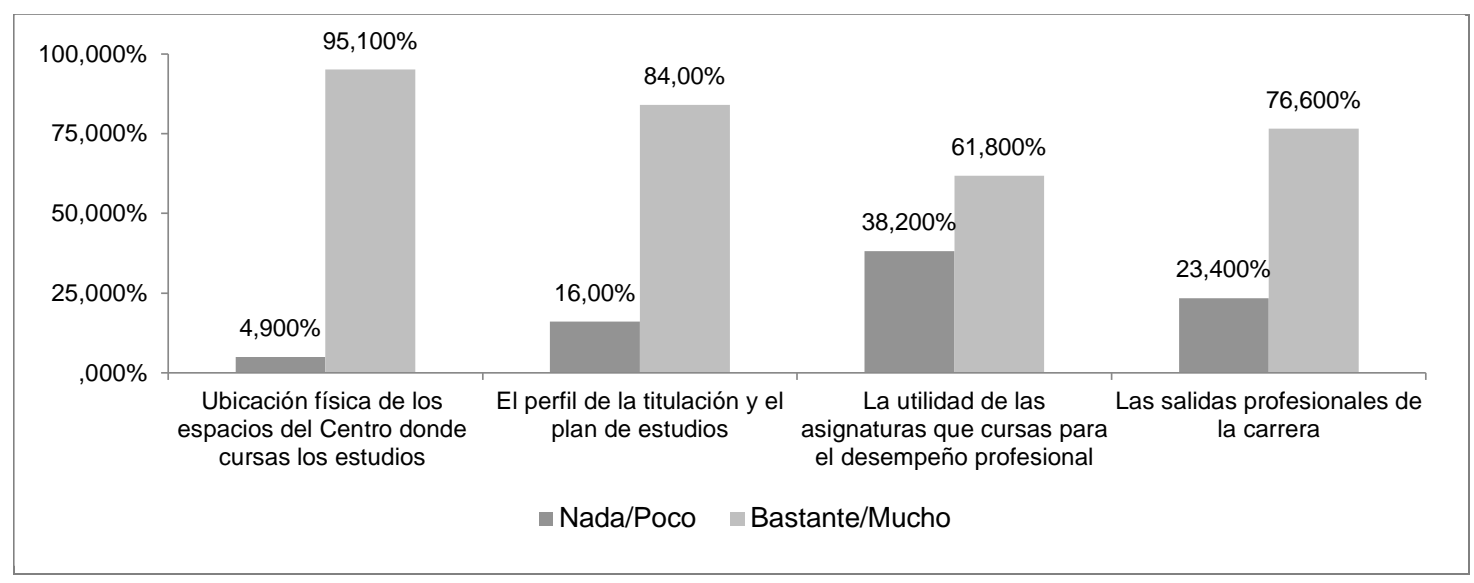

\section{Apoyos y recursos de la universidad}

Finalmente, y en cuanto a los apoyos y recursos con los que contaba la universidad para atender a la formación de los estudiantes deportistas, éstos entendían que los servicios con los que contaban no eran ni adecuados ni suficientes para dar respuesta a sus necesidades $(84,8 \%)$. 
Respecto a los apoyos específicos que ofrecía la universidad para compensar las dificultades formativas que derivan de la armonización entre la práctica deportiva y los estudios, la mayoría de los estudiantes destacaron la importancia de los programas de tutorías personalizadas para atender a sus necesidades académicas y deportivas (95,1\%). En este sentido, los deportistas resaltaron la necesidad de contar con un tutor personal que mediara entre los estudiantes y el profesorado de las distintas materias. Concretamente, el tutor debía ayudar a resolver problemas específicos de su formación (91,4\%), ofrecer orientaciones sobre diferentes procedimientos administrativos (91,4\%), ayudar a resolver dudas sobre sus estudios $(87,6 \%)$ y colaborar en la construcción de su proyecto formativo $(82,7 \%)$ y profesional $(87,3 \%)$.

Respecto al apoyo ofrecido por el profesorado para atender las necesidades educativas de los deportistas, los estudiantes solicitaban cambios en las fechas de los exámenes (54,7\%) y justificación de las faltas de asistencia a clase por motivos deportivos (56,0\%). A juicio del alumnado encuestado, el profesorado no realizaba estas adaptaciones académicas para facilitar el ajuste de tiempo entre práctica deportiva y actividades formativas. Además, los estudiantes demandaron un seguimiento personalizado por parte del profesorado $(83,3 \%)$, la adecuación de la enseñanza a las condiciones formativas propias de los deportistas $(68,8 \%)$ y estrategias metodológicas para dar respuesta a la diversidad y propiciar la inclusión en las aulas universitarias $(75,0 \%)$.

\section{FIGURA 5. Adaptaciones realizadas por el profesorado para adaptarse a las necesidades de los estudiantes}

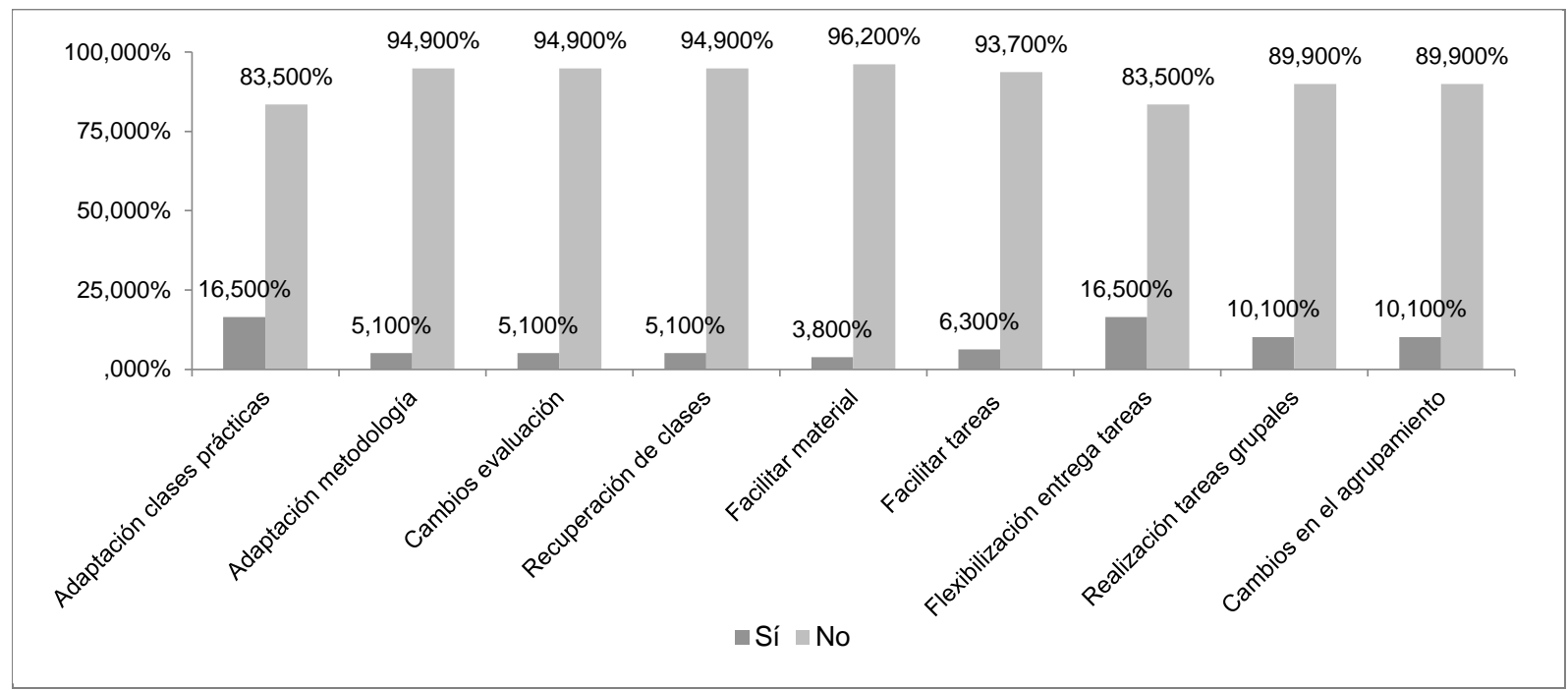

De los datos se desprende también la importancia atribuida a los compañeros y compañeras como agentes de apoyo al proceso formativo de los deportistas de alto nivel, quienes facilitaban la armonización entre el aprendizaje y la práctica deportiva, ofreciendo su ayuda mediante la administración de apuntes e información sobre el avance de las clases (89,9\%). 


\section{Conclusiones}

La investigación desarrollada aporta información relevante sobre cómo llevan a cabo de forma paralela los estudios y la actividad deportiva los estudiantes universitarios deportistas de alto nivel, qué dificultades encuentran para armonizar ambos procesos y qué demandan para un mejor abordaje conjunto de ambas actividades. Como en otros estudios que han tratado la compleja relación entre estudios y deporte (Márquez, Salguero y Tuero, 2003; Puig y Vilanova, 2006), también aquí se ha puesto de manifiesto que los estudiantes atribuyen un alto valor a ambos procesos, ya que forman parte de su desarrollo integral, tanto en el plano personal como profesional. Sin embargo, las dificultades para articular ambos procesos son diversas y tienen que ver con la falta de tiempo, la falta de información y orientación recibida, las dificultades para planificar las distintas tareas, la falta de estrategias para afrontar el proceso de estudio autónomo, las dificultades para mantener las expectativas de logro, las dificultades para cumplir con las tareas académicas, etc. De todos estos factores y al igual que se destaca en el estudio realizado por Macarro, Romero y Torres (2010), la falta de tiempo para atender los requisitos del proceso de aprendizaje constituye uno de los grandes inconvenientes con el que tropiezan los estudiantes que practican deporte de alta competición.

En relación a los objetivos planteados en el estudio, se confirma que el acceso y adaptación de los estudiantes deportistas de alto nivel a la enseñanza universitaria es compleja. A pesar de que los resultados evidencian que los estudiantes deportistas de alto nivel eligieron la titulación universitaria en función de sus intereses vocacionales y por las salidas profesionales que ofrecía la misma, esto no les garantiza el éxito académico. Las circunstancias que rodean el proceso formativo de estos estudiantes, hacen que las variables del sujeto y las características personales tengan un enorme peso en las posibilidades de adaptación y logro de los objetivos académicos. Como se ha señalado en otros estudios (Álvarez, Cabrera, González y Bethencourt, 2006; Álvarez, Figuera y Torrado, 2011), una buena integración a la educación superior pasa por ser persistente, constante, poseer capacidad de toma de decisiones y de planificación de tiempo y objetivos. Sin el dominio de estas competencias genéricas, cuyo desarrollo se debería promover de forma continua a lo largo de todas las etapas educativas, muchos estudiantes deportistas de alto nivel podrían tener dificultades para mantenerse y concluir los estudios superiores. En este caso, los resultados de la investigación revelaron claramente que los estudiantes tenían un alto nivel de motivación por los estudios universitarios, lo cual sin duda se puede considerar un factor de peso que ayude y contribuya al logro de sus metas, pero sería necesario desarrollar actuaciones para un desarrollo integral que englobe, tanto la formación académica, como una formación en competencias transversales.

En cuanto a las necesidades de orientación, los datos obtenidos indican que el alumnado deportista de alto nivel demanda más ayuda, asesoramiento e información en las etapas previas, para poder afrontar el tránsito a la enseñanza universitaria. En este sentido, es significativa la escasa relevancia que para estos estudiantes tuvieron las actividades de orientación en las etapa preuniversitaria y el escaso interés que para ellos tuvo el trabajo realizado por los orientadores y profesores tutores del bachillerato, a la hora de ayudarles a clarificar y preparar el acceso a la universidad. Los estudiantes señalaron que en el momento de acceder a los estudios superiores no tenían información del plan de estudios de la titulación que iban a realizar y de las características del modelo formativo, en cuanto a metodología, sistemas de evaluación o sistemas de apoyo al alumnado. Sin información, sin orientación y sin ayuda, el cambio entre etapas educativas y, en este caso, el acceso a la formación universitaria por parte de estos estudiantes, se convierte en un proceso complejo. Como señalaba Kirton (2000), la falta de percepción del ambiente universitario y la autoeficacia académica, constituyen factores de mucha influencia en la adaptación y persistencia en los estudios a lo largo del primer año. Y en el caso de los participantes de este estudio, es evidente que la información que recibieron sobre los servicios, 
recursos específicos ofrecidos por la universidad para los deportistas de alto nivel y las ayudas y becas disponibles fue deficitaria.

Respecto a los apoyos y ayudas recibidas desde el momento en que se integraron a la enseñanza universitaria, los estudiantes destacaron que la universidad debe mejorar los servicios y las medidas de atención al alumnado deportista de alto nivel. Atendiendo a los resultados, se ve la necesidad de mejorar el grado de implicación del profesorado para dar respuesta a las necesidades específicas de este tipo de alumnado, flexibilizando los procesos instructivos y llevando a cabo las adaptaciones curriculares necesarias para que puedan compaginar estudios y práctica deportiva. Sobre todo cabe destacar las demandas que plantean los estudiantes deportistas de alto nivel en relación a tres factores claves: la necesidad de llevar a cabo un seguimiento más personalizado, la necesidad de realizar adaptaciones en los procesos educativos para dar una respuesta a la situación particular de estos estudiantes y la introducción de recursos y estrategias que faciliten la participación y la continuidad en el proceso de aprendizaje. En general, los datos obtenidos nos llevan a la conclusión de que, para los estudiantes deportistas de alto nivel, el profesorado no había llevado a cabo una atención específica adecuada y adaptada a sus necesidades específicas.

Los resultados de este estudio dan pie para plantear la puesta en práctica de programas de tutoría a través de los cuales se dé una respuesta adaptada a las necesidades de estos estudiantes. En la línea de los planteamientos que realizan Braxton y McClendon (2002) y Álvarez (2012), deberían implantarse en la enseñanza universitaria acciones específicas encaminadas a facilitar la adaptación y retención de los estudiantes deportistas de alto nivel. Las medidas a adoptar tendrían que dirigirse a la mejora del asesoramiento académico, las políticas universitarias, los recursos e infraestructuras, la captación de estudiantes, la formación del profesorado, el reconocimiento de la labor asesora del profesorado y los programas de información, orientación y tutoría. A nivel académico y pensando en las necesidades de los estudiantes deportistas de alto nivel, un sistema de tutoría personalizada podría ser una estrategia adecuada para que el profesor tutor, a lo largo de la titulación, les asesore, les acompañe y les ayude a definir su proyecto formativo y profesional, colaborando directamente con ellos y mediando con otros profesionales para la resolución de los diferentes problemas a los que se enfrentan a lo largo del proceso formativo.

\section{Referencias bibliográficas}

Álvarez, M. (2008). La tutoría académica en el Espacio Europeo de la Educación Superior. Revista Interuniversitaria de Formación del Profesorado, 22, 77-88.

Álvarez, M., Figuera, P. y Torrado, M. (2011). La problemática de la transición BachilleratoUniversidad en la Universidad de Barcelona. REOP, 22 (1), 15-27.

Álvarez, P. (2002). La función tutorial en la universidad; una apuesta por la mejora de la calidad de la enseñanza. Madrid: EOS.

Álvarez, P; Cabrera, L.; González, M; Bethencourt, J. (2006). Causas del abandono y prolongación de los estudios universitarios. Paradigma, 27 (1), 7-36.

Álvarez, P. (coord.) (2012). Tutoría universitaria inclusiva: guía de buenas prácticas para la orientación de estudiantes con necesidades educativas específicas. Madrid: Narcea.

Álvarez, P.; Alegre, O.M. y López, D. (2012). Las dificultades de adaptación a la enseñanza universitaria de los estudiantes con discapacidad: un análisis desde un enfoque de 
orientación inclusiva. RELIEVE, Revista Electrónica de Investigación y Evaluación Educativa, 18 (2).

Álvarez, V. y Lázaro, A. (2002). Calidad de las universidades y orientación universitaria. Málaga: Aljibe.

Arnaiz, P. (2002). Educar en y para la diversidad. En Soto Pérez, F. y López Navarro, J. Nuevas Tecnologías, Viejas Esperanzas: las nuevas tecnologías en el ámbito de la discapacidad y las necesidades especiales. Murcia: Consejería de Educación y Cultura.

Benito, A y Cruz, A. (2005). Nuevas claves para la docencia universitaria en el Espacio Europeo de Educación Superior. Madrid: Narcea.

Blanco, A. (2009). Desarrollo y evaluación de competencias en Educación Superior. Madrid: Narcea.

Braxton, J.M., \& McClendon, S.A. (2002). The fostering of social integration through institutional practice. Journal of College Student Retention: Research, Theory and Practice, 3 (1), 57-71.

Campos, M.C., Ríes, F. y Castillo, O. (2011). Análisis de las competencias adquiridas y utilizadas por los egresados maestros en Educación Física. Revista Internacional de Ciencias del Deporte, 24 (7), 216-229.

Corominas, E. (2001). Competencias genéricas en la formación universitaria. Revista de Educación, 325, 299-321.

Gadamer, H. (2000). La educación es educarse. Paidós: Ibérica.

García, M. (2006). Las competencias de los alumnos universitarios. Revista interuniversitaria de Formación de Profesorado, 20 (3), 253-269.

González, M.; Álvarez, P.; Cabrera, L. y Bethencourt, J. (2007). El abandono de los estudios universitarios: factores determinantes y medidas preventivas. Revista Española de Pedagogía, LXV (236), 71-85.

Kirton, M.J. (2000). Transitional factors influencing the academic persistence of first semester undergraduate freshmen. Dissertation Abstracts International Section A: Humanities \& Social Sciences, 61 (2-A), 522.

Macarro, J.; Romero, C. y Torres, J. (2009). Motivos de abandono de la práctica de actividades físico-deportivas en los estudiantes de bachillerato de la provincia de Granada. Revista de Educación, 353, 495-51.

Márquez, S.; Salguero, A. y Tuero, C. (2003). Adaptación española del cuestionario de causas de abandono en la práctica deportiva: validación y diferencias de género en jóvenes nadadores. Revista Digital, 8 (56).

Martínez, M. (2009). La Orientación y la tutoría en la universidad en el marco del Espacio Europeo de Educación Superior (EEES). Revista Fuentes, 9, 78-97.

Morillas, M.; Gamito, J.; Gómez, J.M.; Sánchez, J.E. y Valiente, M. (2004). Estudio de las dificultades que encuentran los deportistas jóvenes de élite en el desarrollo de su vida personal, social, académica y deportivo-competitiva. Revista Digital, 10 (74). Consultado el 22/04/2013 en: http://www.efdeportes.com/efd74/elite.htm

OCDE (2005). The definition and Selection of key Competencies. Executive Summary. París: OCDE.

Palou, P.; Ponsenti, X.; Gili, M.; Borras, P.A. y Vidal, J. (2005). Motivos para el inicio, mantenimiento y abandono de la práctica deportiva de los preadolescentes de la isla de Mallorca. Ciencias aplicadas a la actividad física y el deporte, 81, 5-11. 
Poblete, M. y col. (2007). Desarrollo de competencias y créditos transferibles. Experiencia multidisciplinar en el contexto universitario. Bilbao: Mensajero.

Puig, N. y Vilanova, A. (2006). Deportistas olímpicos y estrategias de inserción laboral. Revista Internacional de Sociología, LXIV (44).

Real Decreto 1044/2004, de 1 de agosto, por el que se establece el procedimiento para la expedición por las universidades del Suplemento Europeo al Título.

Real Decreto 22/2005, de 21 de enero, por el que se establece la estructura de las enseñanzas universitarias y se regulan los estudios universitarios oficiales de grado.

Real Decreto 1393/2007, de 29 de octubre, por el que se establece la ordenación de las enseñanzas universitarias oficiales.

Real Decreto 861/2010, de 2 de julio, por el que se modifica el Real Decreto 1393/2007, de 29 de octubre, por el que se establece la ordenación de las enseñanzas universitarias oficiales.

Real Decreto 1791/2010, de 30 de diciembre, por el que se aprueba el Estatuto del Estudiante Universitario.

Riesgo, M. (2008). El enfoque por competencias en el EEES y sus implicaciones en la enseñanza y el aprendizaje. Tendencias Pedagógicas 13, 79-105.

Rodríguez, A. (2007). Las competencias en el Espacio Europeo de Educación Superior: Tipologías. Humanismo y Trabajo Social, 6, 83-91.

Rodríguez, S. (coord.) (2004). Manual de tutoría universitaria. Barcelona: Octaedro-ICE.

Rodríguez, S.; Fita, E. y Torrado, M. (2004) El rendimiento académico en la transición secundariauniversidad. Revista de Educación, 334, 391-414.

Rychen, D. y Salganik, L. (2001). Defining and selecting key competencias. Germany: Gotingen, Hogrefe and Huber.

Sánchez, M.F. (2001). La orientación universitaria y las circunstancias de elección de los estudios. Revista de Investigación Educativa, 19 (1), 39-61.

Sánchez, P. y Zubillaga, A. (2005). Las universidades españolas ante el proceso de Convergencia Europeo: análisis de las medidas institucionales y acciones de aplicación y coordinación. Revista de Educación, 337, 160-187.

Unesco (2005). Guidelines for inclusion: ensuring access to education for all. París.

Villa, A. y Bezanilla, Ma (2002). El desarrollo de las competencias en la formación universitaria. Inédito, Bilbao: Universidad de Deusto.

Zabalza, M.A. (2002). La enseñanza universitaria. El escenario y sus protagonistas. Madrid: Narcea.

Fecha de entrada: 25 de Junio de 2013

Fecha de revisión: 11 de Julio de 2013

Fecha de aceptación: 1 de Diciembre de 2013 